\title{
ON THE CONTROL OF AN EVOLUTIONARY EQUILIBRIUM IN MICROMAGNETICS
}

\author{
By
}

\author{
Michal Kočvara \\ Martin Kružík \\ and \\ Jiří V. Outrata
}

IMA Preprint Series \# 2026

( February 2005)

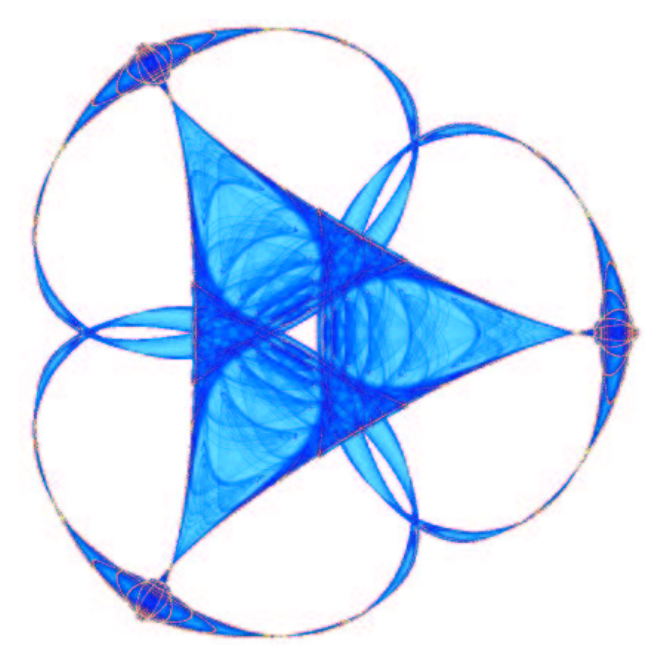

INSTITUTE FOR MATHEMATICS AND ITS APPLICATIONS

UNIVERSITY OF MINNESOTA

514 Vincent Hall

206 Church Street S.E.

Minneapolis, Minnesota 55455-0436

Phone: 612/624-6066 Fax: 612/626-7370

URL: http://www.ima.umn.edu 


\title{
On the control of an evolutionary equilibrium in micromagnetics
}

\author{
Michal Kočvara, Martin Kružík \\ Jiří V. Outrata \\ Institute of Information Theory and Automation \\ Academy of Sciences of the Czech Republic \\ Pod vodárenskou věží 4, CZ-182 08 Praha 8, Czech Republic
}

February 16, 2005

\begin{abstract}
Optimal control of magnetization in a ferromagnet is formulated as a mathematical program with evolutionary equilibrium constraints. To this purpose, we construct an evolutionary infinite-dimensional model which is discretized both in the space as well as in time variables. The evolutionary nature of this equilibrium is due to the hysteresis behavior of the respective magnetization process. To solve the problem numerically, we adapted the implicit programming technique. The adjoint equations, needed to compute subgradients of the composite objective, are derived using the generalized differential calculus of B. Mordukhovich. We solve two test examples and discuss obtained numerical results.
\end{abstract}

Mathematical Subject Classification (2000): 35Q60, 49J, 78M30, 90C26

Keywords: coderivatives, ferromagnetism, hysteresis, implicit programming technique, mathematical programs with equilibrium constraints

\section{Introduction}

In connection with the study of a delamination process in [9], an evolutionary equilibrium model has been introduced which takes into account the irreversibility of changes occurring during the process. In this model, after a suitable discretization, one gets a sequence of optimization problems, where the solution of the $i$ th problem enters the $(i+1)$ th problem as a parameter. In [10], two of the 
authors studied an optimization problem, in which such an evolutionary equilibrium arises among the constraints. Using the generalized differential calculus of B. Mordukhovich, they derived first order optimality conditions. Unfortunately, due to multiplicity of equilibria (which is typical in delamination), they were not able to propose any reasonable procedure to the numerical solution of the investigated problem. It seems, however, that evolutionary equilibrium models can emerge from another application areas, where the uniqueness of equilibria can be ensured. This is, for instance, the case of the model from [14, 34] describing the magnetization of a piece of material in an external magnetic field. This model, based on a detailed study of magnetic microstructures of the considered material, leads to a sequence of coupled optimization problems which are convex, nonsmooth but, in contrast to the delamination, uniqueness of their solutions can be enforced. One can consider the external magnetic field as a control variable and think about an "optimal" magnetization of the sample at the given terminal time. This problem is similar to the control of delamination studied in [10] so that the respective optimality conditions can be derived exactly in the same way. Moreover, due to the uniqueness of equilibria, an effective method to the numerical solution of this problem can be proposed.

The aim of our paper is

- to adopt the so-called implicit programming approach $(\operatorname{ImP})$, analyzed thoroughly in $[17,26,3,11]$ to optimization problems with (locally) uniquely solvable evolutionary equilibria among the constraints, and

- to apply the proposed technique to a concrete problem, where the considered equilibrium describes the above mentioned magnetization.

The structure of the paper is as follows. In Section 2 we develop a variant of $\mathrm{ImP}$ for evolutionary equilibria. This equilibrium is thoroughly studied in Section 3. We start with the original infinite-dimensional formulation, construct a suitable discretization and investigate the relevant properties. Section 4 then deals with an application of the results obtained in Section 2 to the discretized equilibrium derived in Section 3. This section also contains results of numerical experiments.

Our notation is basically standard. The unit matrix is denoted by $\mathbb{I}$ and lin $a$ is the linear hull of a vector $a$. If $f$ is a differentiable function of three variables, then $\nabla_{i} f$ means its gradient (Jacobian) with respect to the $i$ th variable, $i=1,2,3$. For a multifunction $\Phi: \mathbb{R}^{p} \rightsquigarrow \mathbb{R}^{q}$, Gph $\Phi=\left\{(x, y) \in \mathbb{R}^{p} \times \mathbb{R}^{q} \mid y \in \Phi(x)\right\}$ and for a function $f: \mathbb{R}^{p} \rightarrow \overline{\mathbb{R}}$, epi $f=\left\{(x, r) \in \mathbb{R}^{p} \times \mathbb{R} \mid r \geq f(x)\right\}$. If $A$ is an $m \times n$ matrix and $J \subset\{1,2, \ldots, n\}$, then $A_{J}$ is the submatrix of $A$, whose columns are specified by $J$. Similarly, for a vector $d \in \mathbb{R}^{n}, d_{J}$ is a subvector composed from the components specified by $J$. In Sections 3 and 4 we work with vectors having the structure $d=\left(d^{1}, \ldots, d^{s}\right)$, where $d^{i} \in \mathbb{R}^{l}, i=1, \ldots, s$. Then $(d)^{j}$ is the $j$ th 
component of the whole vector $d$, whereas $d^{i j}$ means the $j$ th component of the subvector $d^{i}$. Finally, $o: \mathbb{R}_{+} \rightarrow \mathbb{R}$ is a function such that $\lim _{\lambda \rightarrow 0} o(\lambda) / \lambda=0$.

For reader's convenience, we recall definitions of basic notions from the generalized differential calculus of B. Mordukhovich that will be extensively used in the sequel.

Consider a closed set $\Pi \subset \mathbb{R}^{p}$.

Definition 1.1 Let $\bar{a} \in \Pi$.

(i) The Fréchet normal cone to $\Pi$ at $\bar{a}$, denoted $\widehat{N}_{\Pi}(\bar{a})$, is given by

$$
\widehat{N}_{\Pi}(\bar{a})=\left\{v \in \mathbb{R}^{p} \mid\langle v, a-\bar{a}\rangle \leq o(\|a-\bar{a}\|) \quad \text { for } a \in \Pi\right\} .
$$

(ii) The limiting normal cone to $\Pi$ at $\bar{a}$, denoted $N_{\Pi}(\bar{a})$, is given by

$$
N_{\Pi}(\bar{a}):=\limsup _{a \stackrel{\Pi}{\longrightarrow}_{\bar{a}}} \widehat{N}_{\Pi}(a),
$$

where "limsup" is the upper limit of multifunctions in the sense of KuratowskiPainlevé ([1]) and $a \stackrel{\Pi}{\longrightarrow} \bar{a}$ means $a \rightarrow \bar{a}$ with $a \in \Pi$.

If $N_{\Pi}(\bar{a})=\hat{N}_{\Pi}(\bar{a})$, we say that $\Pi$ is normally regular at $\bar{a}$. Each convex set is normally regular at all its points and $N_{\Pi}$ amounts then to the classic normal cone from convex analysis. In general, however, $N_{\Pi}(\bar{a})$ is nonconvex, but the multifunction $N_{\Pi}(\cdot)$ is upper semicontinuous at each point $\Pi$ (with respect to $\Pi$ ). The local behavior of (extended) real-valued functions and multifunctions is described by subdifferentials and coderivatives defined next.

Definition 1.2 Let $\varphi: \mathbb{R}^{p} \rightarrow \overline{\mathbb{R}}$ be an arbitrary extended real-valued function and $a \in \operatorname{dom} \varphi$. The set

$$
\partial \varphi(a):=\left\{\alpha \in \mathbb{R}^{p} \mid(\alpha,-1) \in N_{\mathrm{epi} \varphi}(a, \varphi(a))\right\}
$$

is called the limiting subdifferential of $\varphi$ at a.

Definition 1.3 Let $\Phi: \mathbb{R}^{p} \rightsquigarrow \mathbb{R}^{q}$ be an arbitrary multifunction with a closed graph and $(a, b) \in \mathrm{Gph} \Phi$.

(i) The multifunction $\widehat{D}^{*} \Phi(a, b): \mathbb{R}^{q} \rightsquigarrow \mathbb{R}^{p}$ defined by

$$
\widehat{D}^{*} \Phi(a, b)(v):=\left\{u \in \mathbb{R}^{p} \mid(u,-v) \in \widehat{N}_{\mathrm{Gph} \Phi}(a, b)\right\}, \quad v \in \mathbb{R}^{q}
$$

is called the regular coderivative of $\Phi$ at $(a, b)$.

(ii) The multifunction $D^{*} \Phi(a, b): \mathbb{R}^{q} \rightsquigarrow \mathbb{R}^{p}$ defined by

$$
D^{*} \Phi(a, b)(v):=\left\{u \in \mathbb{R}^{p} \mid(u,-v) \in N_{\mathrm{Gph} \Phi}(a, b)\right\}, \quad v \in \mathbb{R}^{q}
$$

is called the coderivative of $\Phi$ at $(a, b)$.

If $\Phi$ is single-valued, we write simply $\widehat{D}^{*} \Phi(a)(v)$ and $D^{*} \Phi(a)(v)$.

The interested reader is referred, e.g., to [24] and [31] where the properties of the above objects are studied in detail. 


\section{Problem formulation and implicit program- ming}

In accordance with the literature, under mathematical program with equilibrium constraints (MPEC) we understand the optimization problem

$$
\begin{aligned}
\operatorname{minimize} & \varphi(x, y) \\
\text { subject to } & \\
& y \in S(x) \\
& (x, y) \in \kappa,
\end{aligned}
$$

where $x \in \mathbb{R}^{n}$ is the control or design variable, $y \in \mathbb{R}^{m}$ is the state variable, the multifunction $S: \mathbb{R} \rightsquigarrow \mathbb{R}^{m}$ assigns $x$ a (possibly empty) set of solutions to an equilibrium problem and $\kappa \subset \mathbb{R}^{n} \times \mathbb{R}^{m}$ comprises all "nonequilibrial" constraints. $S$ is usually defined by a generalized equation (GE) which may attain, e.g., the form

$$
0 \in F(x, y)+Q(y)
$$

In (2.2), $F: \mathbb{R}^{n} \times \mathbb{R}^{m} \rightarrow \mathbb{R}^{m}$ is a continuously differentiable operator, whereas $Q: \mathbb{R}^{m} \rightsquigarrow \mathbb{R}^{m}$ is a multifunction with a closed graph. In the formulation of an evolutionary equilibrium one usually has a finite process time interval. After the time discretization, we will thus be dealing with $T \in \mathbb{N}$ time instants uniformly distributed over this interval. Put $m=k T$ with a positive integer $k$ and $y=$ $\left(y_{1}, y_{2}, \ldots, y_{T}\right) \in\left(\mathbb{R}^{k}\right)^{T}$. The "state map" $S$ is now given by the sequence of GEs

$$
0 \in F_{i}\left(x, y_{i-1}, y_{i}\right)+Q_{i}\left(y_{i-1}, y_{i}\right), \quad i=1,2, \ldots, T
$$

with a given initial state $y_{0}$. In (2.3) the maps $F_{i}: \mathbb{R}^{n} \times \mathbb{R}^{k} \times \mathbb{R}^{k} \rightarrow \mathbb{R}^{k}$ are continuously differentiable and $Q_{i}: \mathbb{R}^{k} \times \mathbb{R}^{k} \rightsquigarrow \mathbb{R}^{k}$ are closed-graph multifunctions. We will call (2.1) the mathematical program with evolutionary equilibrium constraints and use the acronym MPEEC. Similarly as in [10], we confine ourselves to the case, where $\varphi$ depends only on $y_{T}$, the terminal component of $y$. In contrast to [10], however, we now concentrate on the numerical solution of MPEEC. To this purpose, we impose the following simplifying assumptions:

A1: $\varphi$ is continuously differentiable.

A2: $\kappa=\omega \times \mathbb{R}^{m}$, where $\omega \subset \mathbb{R}^{n}$ is a nonempty closed set of admissible controls.

A3: The state map $S$ (defined via GEs (2.3)) is single-valued and locally Lipschitz on an open set containing $\omega$.

While assumption (A1) is not too restrictive for many applications, assumption (A2) complicates the treatment of possible state or mixed state-control constraints which have to be handled via a smooth penalty. The most restrictive 
is, however, assumption (A3). In fact, this assumption can be replaced by the requirement that $S$ possesses a Lipschitz single-valued localization at each pair $(x, y), x \in \omega, y \in S(x)$; see [31]. Such a weakened variant can be verified by using the concept of strong regularity due to Robinson [29]. We return to the question of the verification of (A3) later.

The key idea of implicit programming ( $\mathrm{ImP}$ ) consists in the reformulation of (2.1) to the form

$$
\begin{aligned}
\operatorname{minimize} & \Theta(x) \\
\text { subject to } & \\
& x \in \omega,
\end{aligned}
$$

where $\Theta(x):=\varphi\left(x, S_{T}(x)\right)$, and $S_{T}$ assigns $x$ the respective terminal state $y_{T}$. Due to (A1), (A3), $\Theta$ is locally Lipschitz on an open set containing $\omega$ and so various methods can be used to the numerical solution of (2.4); see [17],[26]. In this paper, we apply a bundle method of nonsmooth optimization; in particular, the BT algorithm described in [36]. This means that we must be able to compute for each admissible control $x$ at least one arbitrary subgradient $\xi$ from $\bar{\partial} \Theta(x)$, the Clarke subdifferential of $\Theta$ at $x$. If $S$ happens to be a $P C^{1}$ map (see [35]), one can apply the classic implicit function theorem to an essentially active component of $S$ and arrives in this way at a desired subgradient $\xi$. This technique has been used in [26]. In [11], a different approach is suggested based on the generalized differential calculus of Mordukhovich. This approach is not restricted to $P C^{1}$ state maps and we will apply it also in the case of our evolutionary equilibrium. To simplify the notation, let $\widetilde{Q}_{1}\left(y_{1}\right):=Q_{1}\left(y_{0}, y_{1}\right)$.

Theorem 2.1 Let assumptions (A1)-(A3) hold true, $\bar{x} \in \omega, \bar{y}=$ $\left(\bar{y}_{1}, \bar{y}_{2}, \ldots, \bar{y}_{T}\right)=S(\bar{x})$, and $\bar{c}_{i}:=-F_{i}\left(\bar{x}, \bar{y}_{i-1}, \bar{y}_{i}\right), i=1,2, \ldots, T$, with $\bar{y}_{0}:=y_{0}$. Assume that the three sequences of adjoint vectors $q_{2}, q_{3}, \ldots, q_{T}, v_{1}, v_{2}, \ldots, v_{T}$, $w_{1}, w_{2}, \ldots, w_{T}$ fulfill the relations

$$
\begin{aligned}
v_{1} & \in \widehat{D}^{*} \widetilde{Q}_{1}\left(\bar{y}_{1}, \bar{c}_{1}\right)\left(w_{1}\right) \\
\left(q_{i}, v_{i}\right) & \in \widehat{D}^{*} Q_{i}\left(\bar{y}_{i-1}, \bar{y}_{i}, \bar{c}_{i}\right)\left(w_{i}\right), \quad i=2,3, \ldots, T,
\end{aligned}
$$

and satisfy the adjoint system

$$
\begin{aligned}
& 0=\nabla_{2} \varphi\left(\bar{x}, \bar{y}_{T}\right)+\left(\nabla_{3} F_{T}\left(\bar{x}, \bar{y}_{T-1}, \bar{y}_{T}\right)\right)^{T} w_{T}+v_{T} \\
& 0=\left(\nabla_{3} F_{T-1}\left(\bar{x}, \bar{y}_{T-2}, \bar{y}_{T-1}\right)\right)^{T} w_{T-1}+v_{T-1} \\
& +\left(\nabla_{2} F_{T}\left(\bar{x}, \bar{y}_{T-1}, \bar{y}_{T}\right)\right)^{T} w_{T}+q_{T} \\
& 0=\left(\nabla_{3} F_{1}\left(\bar{x}, \bar{y}_{0}, \bar{y}_{1}\right)\right)^{T} w_{1}+v_{1}+\left(\nabla_{2} F_{2}\left(\bar{x}, \bar{y}_{1}, \bar{y}_{2}\right)\right)^{T} w_{2}+q_{2} .
\end{aligned}
$$


Then one has

$$
\xi:=\nabla_{1} \varphi\left(\bar{x}, \bar{y}_{T}\right)+\sum_{i=1}^{T}\left(\nabla_{1} F_{i}\left(\bar{x}, \bar{y}_{i-1}, \bar{y}_{i}\right)\right)^{T} w_{i} \in \bar{\partial} \Theta(\bar{x}) .
$$

Proof. Let $b$ denote the vector

$$
\left(0,0, \ldots, \nabla_{2} \varphi\left(\bar{x}, \bar{y}_{T}\right)\right) \in\left(\mathbb{R}^{k}\right)^{T} .
$$

From [31, Thm.10.49] it follows that

$$
\begin{aligned}
\bar{\partial} \Theta(\bar{x}) \supset \partial \Theta(\bar{x}) \supset \nabla_{1} \varphi\left(\bar{x}, \bar{y}_{T}\right)+\widehat{D}^{*} S_{T}\left(\bar{x}, \bar{y}_{T}\right)\left(\nabla_{2} \varphi\left(\bar{x}, \bar{y}_{T}\right)\right) & \\
& =\nabla_{1} \varphi\left(\bar{x}, \bar{y}_{T}\right)+\widehat{D}^{*} S(\bar{x}, \bar{y})(b) .
\end{aligned}
$$

Further, one observes that

$$
S(x)=\left\{y \in \mathbb{R}^{k T} \mid \Phi(x, y) \in \Lambda\right\},
$$

where

$$
\Phi(x, y)=\left[\begin{array}{c}
y_{1} \\
-F_{1}\left(x, y_{0}, y_{1}\right) \\
y_{1} \\
y_{2} \\
-F_{2}\left(x, y_{1}, y_{2}\right) \\
\cdots \\
y_{T-1} \\
y_{T} \\
-F_{T}\left(x, y_{T-1}, y_{T}\right)
\end{array}\right] \text { and } \Lambda=G p h \widetilde{Q}_{1} \times \underset{i=2}{\mathrm{X}} G p h Q_{i}
$$

By virtue of [31, Thm.6.14],

$$
\widehat{N}_{G p h S}(\bar{x}, \bar{y}) \supset(\nabla \Phi(\bar{x}, \bar{y}))^{T} \widehat{N}_{\Lambda}(\Phi(\bar{x}, \bar{y})),
$$

and thus, by definition,

$$
\widehat{D}^{*} S(\bar{x}, \bar{y})(b) \supset\left\{\left(\nabla_{1} \Phi(\bar{x}, \bar{y})\right)^{T} p \mid-b=\left(\nabla_{2} \Phi(\bar{x}, \bar{y})\right)^{T} p, p \in \widehat{N}_{\Lambda}(\Phi(\bar{x}, \bar{y}))\right\} .
$$

Putting $p=\left(v_{1},-w_{1}, q_{2}, v_{2},-w_{2}, \ldots, q_{T}, v_{T},-w_{T}\right)$, we can now make use of the decomposition

$$
\widehat{N}_{\Lambda}(\Phi(\bar{x}, \bar{y}))=\widehat{N}_{G p h \tilde{Q}_{1}}\left(\bar{x}, \bar{y}_{1}, \bar{c}_{1}\right) \times \stackrel{T}{\mathrm{X}}_{i=2}^{\widehat{N}_{G p h Q_{i}}}\left(\bar{x}, \bar{y}_{i-1}, \bar{y}_{i}, \bar{c}_{i}\right) .
$$

In this way, relation $p \in \widehat{N}_{G p h \Lambda}(\Phi(\bar{x}, \bar{y}))$ implies (2.5), the adjoint system (2.6) amounts exactly to

$$
\left(\nabla_{2} \Phi(\bar{x}, \bar{y})\right)^{T} p+b=0
$$

and formula (2.7) follows from (2.8) and (2.9). 
Unfortunately, even in case of very simple equilibria, it can be rather difficult to fulfill relations (2.5). These relations become, however, substantially easier if we replace the regular coderivatives by (standard) coderivatives. This possibility is examined in the following statement.

Theorem 2.2 Let all assumptions of Theorem 2.1 be fulfilled with relations (2.5) replaced by

$$
\begin{aligned}
v_{1} & \in D^{*} \widetilde{Q}_{1}\left(\bar{y}_{1}, \bar{c}_{1}\right)\left(w_{1}\right) \\
\left(q_{i}, v_{i}\right) & \in D^{*} Q_{i}\left(\bar{y}_{i-1}, \bar{y}_{i}, \bar{c}_{i}\right)\left(w_{i}\right), \quad i=2,3, \ldots, T .
\end{aligned}
$$

Further suppose that the map $\Xi: \mathbb{R}^{3 k T-1} \rightsquigarrow \mathbb{R}^{n} \times \mathbb{R}^{k T}$, defined by

$$
\Xi(z)=\left\{(x, y) \in \mathbb{R}^{n} \times \mathbb{R}^{k T} \mid \Phi(x, y)-z \in \Lambda\right\},
$$

is either polyhedral (see [30]) or possesses the Aubin property around $(0, \bar{x}, \bar{y})$ (see [31, Def. 9.36]). Then one has

$$
\begin{array}{r}
\partial \Theta(\bar{x}) \subset \nabla_{1} \varphi\left(\bar{x}, \bar{y}_{T}\right)+\left\{\sum_{i=1}^{T}\left(\nabla_{1} F_{i}\left(\bar{x}, \bar{y}_{i-1}, \bar{y}_{i}\right)\right)^{T} w_{i} \mid\left(w_{1}, w_{2}, \ldots, w_{T}\right)\right. \\
\text { fulfills relations (2.6),(2.10) with suitable vectors }\left(v_{1}, v_{2}, \ldots, v_{T}\right) \\
\text { and } \left.\left(q_{2}, q_{3}, \ldots, q_{T}\right)\right\}
\end{array}
$$

Inclusion (2.11) becomes equality provided $\widehat{N}_{G p h \widetilde{Q}_{1}}\left(\bar{y}_{1}, \bar{c}_{1}\right)=N_{G p h \widetilde{Q}_{1}}\left(\bar{y}_{1}, \bar{c}_{1}\right)$ and $\widehat{N}_{G p h Q_{i}}\left(\bar{y}_{i-1}, \bar{y}_{i}, \bar{c}_{i}\right)=N_{G p h Q_{i}}\left(\bar{y}_{i-1}, \bar{y}_{i}, \bar{c}_{i}\right), i=2,3, \ldots, T$ (i.e. the graphs of $\widetilde{Q}_{1}$ and $Q_{i}, i=2,3, \ldots, T$, are normally regular at the respective points).

Proof. From [31, Thm. 10.49] it follows that under our assumptions

$$
\partial \Theta(\bar{x}) \subset \nabla_{1} \varphi\left(\bar{x}, \bar{y}_{T}\right)+D^{*} S(\bar{x}, \bar{y})(b) .
$$

Under the assumption imposed on $\Xi$, one has the inclusion

$$
N_{G p h S}(\bar{x}, \bar{y}) \subset(\nabla \Phi(\bar{x}, \bar{y}))^{T} N_{\Lambda}(\Phi(\bar{x}, \bar{y})) ;
$$

see [6, Thm. 4.1],[24, Cor. 5.5]. Thus we can decompose $\Lambda$ in the same way as in the proof of Theorem 2.1 and arrive directly at formula (2.11). Concerning the second assertion, it suffices to compare (2.7) and (2.11) under the imposed regularity of $G p h \widetilde{Q}_{1}$ and $G p h Q_{i}, i=2,3 \ldots, T$.

On the basis of the above analysis, we can now return to the assumption (A3). The single-valuedness of $S$ can be ensured in different ways and in many cases does not represent a serious problem. To enforce the local Lipschitz continuity of $S$, one can require that all functions $F_{i}$ are affine and all multifunctions $Q_{i}$ 
are polyhedral. The result then follows from [30]. Alternatively, by virtue of Theorem 2.3, we can impose the assumption (A4) below at all pairs $(\bar{x}, \bar{y})$, where $\bar{x} \in \omega$ and $\bar{y}=S(\bar{x})$.

Let us call the adjoint system (2.6) homogeneous, provided $\nabla_{2} \varphi(\bar{x}, \bar{y})$ is replaced by the zero vector.

A4: The only vectors $q_{2}, q_{3}, \ldots, q_{T}, v_{2}, v_{3}, \ldots, v_{T}$ and $w_{2}, w_{3}, \ldots, w_{T}$ satisfying (2.10) and the homogeneous adjoint system are the zero vectors.

The role of (A4) is explained in the following statement.

Theorem 2.3 Let $\bar{x} \in \omega, S$ be single-valued around $\bar{x}$ and $\bar{y}=S(\bar{x})$. Assume that (A4) is fulfilled. Then $\Xi$ possesses the Aubin property around $(0, \bar{x}, \bar{y})$ and $S$ is Lipschitz around $\bar{x}$.

Proof. Following [22, Cor. 4.4], we easily infer that the Aubin property of $\Xi$ around $(0, \bar{x}, \bar{y})$ is implied by the requirement

$$
\left.\begin{array}{c}
(\nabla \Phi(\bar{x}, \bar{y}))^{T} p=0 \\
p \in N_{\Lambda}(\Phi(\bar{x}, \bar{y}))
\end{array}\right\} \Rightarrow p=0 .
$$

To ensure the Aubin property of $S$ around $\bar{x}$, we can apply the same result and arrive at the stronger condition

$$
\left.\begin{array}{c}
\left(\nabla_{2} \Phi(\bar{x}, \bar{y})\right)^{T} p=0 \\
p \in N_{\Lambda}(\Phi(\bar{x}, \bar{y}))
\end{array}\right\} \Rightarrow p=0 .
$$

It thus suffices to decompose $\Lambda$ as in the previous statements and observe that (2.12) amounts exactly to (A4). Since $S$ is single-valued on a neighborhood of $\bar{x}$ and possesses the Aubin property around $(\bar{x}, \bar{y})$, it is in fact Lipschitz near $\bar{x}$ and we are done.

Inclusion (2.11) provides us with a desired element $\xi \in \bar{\partial} \Theta(\bar{x})$ only if it becomes equality. This happens in the regular case, mentioned in Theorem 2.2, and also under another additional conditions which will not be discussed here. Fortunately, the used bundle methods converge mostly to a Clarke stationary point even if we replace a vector from $\bar{\partial} \Theta(\bar{x})$ by a vector from the right-hand side of (2.11). Moreover, if some difficulties occur, one can attempt to modify suitably the rules for the selection of vectors $q_{2}, q_{3}, \ldots, q_{T}, v_{1}, v_{2}, \ldots, v_{T}$ and $w_{1}, w_{2}, \ldots, w_{T}$ in (2.10). This remedy will be explained in detail in the last section. To summarize, for reasons of computational complexity, we will supply our bundle methods by vectors coming from the right-hand side of (2.11). It turns out that this way is sufficient for a successful numerical solution of various difficult MPEECs. 
Remark 2.4 In [3] one finds a theory investigating the behavior of a general bundle method, if $\bar{\partial} \Theta$ is replaced by a larger set, satisfying a few reasonable assumptions.

The construction of relations (2.10) and the adjoint system (2.6) will be illustrated in Section 4 by means of an equilibrium model (3.10) derived in the next section.

\section{State problem - hysteresis in micromagnetics}

In this section we describe a hysteresis model in micromagnetics which will be further used for the formulation of an MPEEC. This model was developed in $[13,14,15,33,34]$ and is based on Brown's theory [2] for static ferromagnetism tailored to large specimens by DeSimone [4]. In order to get hysteresis behavior, we enrich the static model by a suitable rate-independent dissipative mechanism. The main difficulty is that the formulation leads to a minimization problem with a nonconvex feasible set. As, in general, the minimum is not attained, one must seek a generalization of the notion "solution". This is done here by means of Young measures $[32,38]$. A formulation of the continuum model is the content of Subsection 3.1. Subsection 3.2 is devoted to a discretization of the problem. We show that the problem leads to a finite sequence of problems having the structure (2.3).

Readers not interested in the physical model and/or in its mathematical treatment may skip this section up to formula (3.13). The main message of this section is that the equilibrium constraint, after a suitable spatial discretization, has the structure (2.3). Also the respective maps $F_{i}, Q_{i}$ are computed.

\subsection{Model}

The theory of rigid ferromagnetic bodies $[2,16,18]$ assumes that a magnetization $M: \Omega \rightarrow \mathbb{R}^{N}$, describing the state of a body $\Omega \subset \mathbb{R}^{N}, N=2,3$, is subjected to the Heisenberg-Weiss constraint, i.e., has a given (in general, temperature dependent) magnitude

$$
|M(z)|=m_{\mathrm{s}} \text { for almost all } z \in \Omega,
$$

where $m_{\mathrm{s}}>0$ is the saturation magnetization, considered here constant.

In the no-exchange formulation, which is valid for large bodies [4], the Helmholtz free energy of a rigid ferromagnetic body $\Omega \subset \mathbb{R}^{N}$ consists of two parts. The first part is the anisotropy energy $\int_{\Omega} \phi(M(z)) \mathrm{d} z$ related to crystallographic properties of the ferromagnet. Denoting $S^{N-1}:=\left\{s \in \mathbb{R}^{N}|| s \mid=m_{\mathrm{s}}\right\}$, a typical $\phi: S^{N-1} \rightarrow \mathbb{R}$ is a nonnegative function vanishing only at a few isolated points on $S^{N-1}$ determining directions of easy magnetization. We are especially 
interested in uniaxial materials (e.g. cobalt), where $\phi$ vanishes exactly at two points. From now on we will assume that the easy axis of the material coincides with the $N$ th coordinate axis.

The second part of the Helmholtz energy, $\frac{1}{2} \int_{\mathbb{R}^{N}}\left|\nabla u_{M}(z)\right|^{2} \mathrm{~d} z$, is the energy of the demagnetizing field $\nabla u_{M}$ self-induced by the magnetization $M$; its potential $u_{M}$ is governed by

$$
\operatorname{div}\left(-\mu_{0} \nabla u_{M}+M \chi_{\Omega}\right)=0 \quad \text { in } \mathbb{R}^{N},
$$

where $\chi_{\Omega}: \mathbb{R}^{N} \rightarrow\{0,1\}$ is the characteristic function of $\Omega$ and $\mu_{0}$ is the vacuum permeability. The demagnetizing-field energy thus penalizes non-divergence-free magnetization vectors. Standardly, we will understand (3.1) in the weak sense, i.e. $u_{M} \in W^{1,2}\left(\mathbb{R}^{N}\right)$ will be called a weak solution to (3.1) if the integral identity $\int_{\mathbb{R}^{N}}\left(M \chi_{\Omega}-\nabla u_{m}(z)\right) \cdot \nabla v(z) \mathrm{d} z=0$ holds for all $v \in W^{1,2}\left(\mathbb{R}^{N}\right)$, where $W^{1,2}\left(\mathbb{R}^{N}\right)$ denotes the Sobolev space of functions in $L^{2}\left(\mathbb{R}^{N}\right)$ with all first derivatives (in the distributional sense) also in $L^{2}\left(\mathbb{R}^{N}\right)$. Altogether, the Helmholtz energy $E(M)$, has the form

$$
E(M)=\int_{\Omega} \phi(M(z)) \mathrm{d} z+\frac{1}{2} \int_{\mathbb{R}^{N}}\left|\nabla u_{M}(z)\right|^{2} \mathrm{~d} z
$$

If the ferromagnetic specimen is exposed to some external magnetic field $h=h(z)$, the so-called Zeeman's energy of interactions between this field and magnetization vectors equals to $H(M):=\int_{\Omega} h(z) \cdot M(z) \mathrm{d} z$. Finally, the following variational principle governs equilibrium configurations:

$$
\begin{aligned}
\operatorname{minimize} & \mathcal{G}(M) \\
& :=E(M)-H(M) \\
& =\int_{\Omega}(\phi(M(z))-h(z) \cdot M(z)) \mathrm{d} z+\frac{1}{2} \int_{\mathbb{R}^{N}}\left|\nabla u_{M}(z)\right|^{2} \mathrm{~d} z
\end{aligned}
$$

subject to

$$
(3.1),\left(M, u_{M}\right) \in \mathcal{A} \times W^{1,2}\left(\mathbb{R}^{N}\right),
$$

where the introduced notation $\mathcal{G}$ stands for Gibbs' energy and $\mathcal{A}$ is the set of admissible magnetizations

$$
\mathcal{A}:=\left\{M \in L^{\infty}\left(\Omega ; \mathbb{R}^{N}\right)|| M(z) \mid=m_{\mathrm{s}} \text { for almost all } z \in \Omega\right\} .
$$

As $\mathcal{A}$ is not convex, we cannot rely on direct methods in proving the existence of a solution. In fact, the solution to (3.3) need not exist in $\mathcal{A} \times W^{1,2}\left(\mathbb{R}^{N}\right)$; see [8] for the uniaxial case. There is a competition of the anisotropy energy in $\mathcal{G}$ preferring the magnetization of the constant length and the demagnetizing field energy preferring it to be zero, which is just what explains quite generic occurrence of the domain microstructure. Mathematically, this is expressed by nonexistence of an exact minimizer of $\mathcal{G}$ and by finer and finer self-similar spatial oscillations necessarily developed in any minimizing sequence of $\mathcal{G}$. 
To pursue evolution in an efficient manner, it is important to collect some information about the fine structure "around" a current point $x \in \Omega$ in the form of a probability measure, denoted by $\nu_{x}$, supported on the sphere $S^{N-1}$. Hence, one has $\nu_{x} \in \mathfrak{M}\left(S^{N-1}\right)$, the set of all probability measures on $S^{N-1}$. Let us furthermore denote by $\mathbb{B}$ the ball $\left\{M \in \mathbb{R}^{N}|| M \mid \leq m_{\mathrm{s}}\right\}$ of the radius $m_{\mathrm{s}}$. The collection $\nu=\left\{\nu_{x}\right\}_{x \in \Omega}$ is often called a Young measure [38, 32] and can be considered a certain "mesoscopic" description of the magnetization. The average, let us call it macroscopic magnetization, $M=M(x)$ at a material point $x \in \Omega$ still remains a worthwhile quantity; it is just the first momentum of the Young measure $\nu=\left\{\nu_{x}\right\}_{x \in \Omega}$, i.e.

$$
M(x)=\int_{S^{N-1}} s \nu_{x}(\mathrm{~d} s) .
$$

Note that the macroscopic magnetization $M: \Omega \rightarrow \mathbb{B}$ "forgets" detailed information about the microstructure in contrast with the mesoscopic magnetization $\nu: \Omega \rightarrow \mathfrak{M}\left(S^{N-1}\right)$ which can capture volume fractions related to particular directions of the magnetization. It should be emphasized that, though we speak about (collections of) probability measures, our approach is fully deterministic.

On the mesoscopic level we write the Gibbs' energy as [28, 32]:

$$
\bar{G}(\nu)=\int_{\Omega}(\phi \bullet \nu-h(z) \cdot M(z)) \mathrm{d} z+\frac{1}{2} \int_{\mathbb{R}^{N}}\left|\nabla u_{M}(z)\right|^{2} \mathrm{~d} z,
$$

where $[v \bullet \nu](z):=\int_{\mathbb{R}^{N}} v(s) \nu_{z}(\mathrm{~d} s)$, id $: \mathbb{R}^{N} \rightarrow \mathbb{R}^{N}$ is the identity, and

$$
M(z)=\int_{S^{N-1}} s \nu_{z}(\mathrm{~d} z)
$$

An important property is that $\bar{G}$ is convex with respect to the natural geometry of probability measures $\nu=\left\{\nu_{z}\right\}_{z \in \Omega}$. Let us denote by $\mathcal{Y}\left(\Omega ; S^{N-1}\right)$ the convex set of families of probability measures in $\mathfrak{M}\left(S^{N-1}\right)$ parameterized by $x \in \Omega$. It is well-known that the minimum of $\bar{G}$ over $\mathcal{Y}\left(\Omega ; S^{N-1}\right)$ is truly attained. We refer to $[4,27,28]$ for a mathematically rigorous reasoning.

We can now model the behavior of low-hysteresis materials with reasonable accuracy by minimizing of $\bar{G}$. Varying the external magnetic field $h$ in time produces, however, only a functional graph in an $h / M$ diagram, but no hysteresis loop. On the other hand, magnetically hard materials, as e.g. CoZrDy, display significant hysteresis and cannot be modeled by mere minimization of $\bar{G}$. Therefore, we must enrich our model and focus thereby on hysteresis losses which are independent of the time frequency of $h$. We refer to [7] for an exposition of various kinds of energy losses in ferromagnets.

Inspired by [37], we describe energetic losses during the magnetization by a phenomenological dissipation potential $\varrho$ depending on the time derivative $\dot{M}$ of the form 


$$
\varrho(\dot{M})=\int_{\Omega} H_{\mathrm{c}}\left|\frac{\mathrm{d} M_{N}}{\mathrm{~d} t}\right| \mathrm{d} z
$$

where the constant $H_{\mathrm{c}}>0$ is the so-called coercive force describing the width of the hysteresis loop and $M_{N}$ is the $N$ th component of the magnetization $M$.

Following [19], we define a dissipation distance $\mathcal{D}$ by

$$
\mathcal{D}(M, \tilde{M}):=\int_{\Omega} H_{\mathrm{c}}\left|M_{N}-\tilde{M}_{N}\right| \mathrm{d} z
$$

Equivalently, this quantity can be written in terms of Young measures as

$$
D(\nu, \tilde{\nu}):=\int_{\Omega} H_{\mathrm{c}}\left|\int_{S^{N-1}} s_{N} \nu_{z}(\mathrm{~d} s)-\int_{S^{N-1}} s_{N} \tilde{\nu}_{z}(\mathrm{~d} s)\right| \mathrm{d} z
$$

where $s=\left(s_{1}, \ldots, s_{N}\right) \in S^{N-1}$. Obviously, the equivalence of (3.7) and (3.8) holds if $M=\mathrm{id} \bullet \nu$ and $\tilde{M}=\mathrm{id} \bullet \tilde{\nu}$. Both the formulas evaluate how much energy is dissipated if we change the magnetization of the specimen from $M$ to $\tilde{M}$. Analogously to $[20,21,34]$ we assume that, having a sequence of discrete time instants, an optimal mesoscopic magnetization at the instant $1 \leq i \leq T$ minimizes $\bar{G}(\nu)+\mathcal{D}\left(M, M_{i-1}\right)$ over $\mathcal{Y}\left(\Omega ; S^{N-1}\right)$ and subject to $(3.6)$, where $\bar{M}_{i-1}$ is the solution at the $(i-1)$ th time step. by

To be more precise, we first define the Gibbs energy at the time $1 \leq i \leq T$,

$$
\bar{G}(i, \nu)=\int_{\Omega}(\phi \bullet \nu-h(i, z) \cdot M(z)) \mathrm{d} z+\frac{1}{2} \int_{\mathbb{R}^{N}}\left|\nabla u_{M}(z)\right|^{2} \mathrm{~d} z
$$

where $h(i, \cdot)$ is an external field at this time. Then, starting with an initial condition $\nu_{0} \in \mathcal{Y}\left(\Omega ; S^{N-1}\right)$, we find consecutively for $i=1, \ldots, T$ a solution $\nu_{i} \in \mathcal{Y}\left(\Omega ; S^{N-1}\right)$ of the minimization problem:

$$
\begin{aligned}
\operatorname{minimize} & I(\nu):=\bar{G}(i, \nu)+D\left(\nu, \nu_{i-1}\right) \\
\text { subject to } & \\
& \nu \in \mathcal{Y}\left(\Omega ; S^{N-1}\right) .
\end{aligned}
$$

Next we are going to show that the solution to (3.10) is unique. The key observation is that the history dependence enters (3.10) only through the first momentum of the Young measure, i.e., through $M_{i-1}$ and that we can rule out the Young measure $\nu$ from the definition of $\bar{G}$ by replacing the term $\int_{\Omega} \phi \bullet \nu \mathrm{d} z$ by $\int_{\Omega} \phi^{* *}\left(M(z) \mathrm{d} z\right.$, with $\phi^{* *}$ being the convex envelope of $\hat{\phi}$, where

$$
\hat{\phi}(m)=\left\{\begin{array}{cc}
\phi(M) & \text { if }|M|=m_{\mathrm{s}} \\
+\infty & \text { otherwise. }
\end{array}\right.
$$


Put

$$
G^{* *}(i, M)=\int_{\Omega}\left(\phi^{* *}(M(z))-h(i, z) \cdot M(z)\right) \mathrm{d} z+\frac{1}{2} \int_{\mathbb{R}^{N}}\left|\nabla u_{M}(z)\right|^{2} \mathrm{~d} z .
$$

Let $M_{0} \in L^{2}\left(\Omega ; \mathbb{R}^{N}\right)$ with $M_{0}(z) \in \mathbb{B}$ for a.a. $z \in \Omega$ be given. We look for a solution $M_{i}, 1 \leq i \leq T$ of the problem

$$
\begin{aligned}
\operatorname{minimize} & I^{* *}(M):=G^{* *}(i, M)+\mathcal{D}\left(M, M_{i-1}\right) \\
\text { subject to } & \\
& M \in L^{2}\left(\Omega ; \mathbb{R}^{N}\right) \text { and } M(z) \in \mathbb{B} \text { for a.a. } z \in \Omega .
\end{aligned}
$$

The problems (3.10) and (3.11) are equivalent in the following sense. If $M_{0}=\mathrm{id} \bullet \nu_{0}$ and if $\nu=\left(\nu_{1}, \ldots, \nu_{T}\right)$ solves (3.10), then $\int_{S^{N-1}} s \nu_{z}(\mathrm{~d} s)$ solves $(3.11)$. Conversely, if $M=\left(M_{0}, \ldots, M_{T}\right)$ solves (3.11) and $\nu_{i} \in \mathcal{Y}\left(\Omega ; S^{N-1}\right), 1 \leq i \leq T$, is such that $M_{i}(z)=\int_{S^{N-1}} s\left(\nu_{i}\right)_{z}(\mathrm{~d} s)$ and $\phi^{* *}\left(M_{i}(z)\right)=\int_{S^{N-1}} \phi(s)\left(\nu^{i}\right)_{z}(\mathrm{~d} s)$ for almost all $x \in \Omega$ and $1 \leq i \leq T$, then $\nu=\left(\nu_{1}, \ldots \nu_{T}\right)$ solves (3.10).

We have the following uniqueness result.

Proposition 3.1 Let $\phi(s)=\gamma \sum_{i=1}^{N-1} s_{i}^{2}, \gamma>0,|s|=m_{\mathrm{s}}$. Then the problem (3.11) has a unique solution.

Proof. Under the assumptions, $\phi^{* *}(s)=\gamma \sum_{i=1}^{N-1} s_{i}^{2}$ for all $s \in \mathbb{R}^{N},|s| \leq m_{\mathrm{s}}$; see [4]. We will proceed by induction.

Suppose that $M_{i-1} \in L^{2}\left(\Omega ; \mathbb{R}^{N}\right), M_{i-1}(z) \in \mathbb{B}$ for a.a. $z \in \Omega$, is given uniquely. Let $\hat{M}$ and $\tilde{M}$ be two different minimizers to $I^{* *}$. Then $\nabla u_{\hat{M}}=\nabla u_{\tilde{M}}$ a.e. in $\mathbb{R}^{N}$. Indeed, if they were different, the convexity $I^{* *}$, the strict convexity of the demagnetizing field energy, i.e. of $\|\cdot\|_{L^{2}\left(\mathbb{R}^{N} ; \mathbb{R}^{N}\right)}^{2}$, and the linearity of the map $M \mapsto \nabla u_{M}$ would give us that $0.5 \hat{M}+0.5 \tilde{M}$ has a strictly lower energy than $I^{* *}(\tilde{M})=I^{* *}(\hat{M})$. Similarly, as $\phi^{* *}$ is strictly convex in the first $(N-1)$ variables, we get that $\tilde{M}_{i}=\hat{M}_{i}$ a.e. in $\Omega$ for $i=1, \ldots, N-1$. Put $\beta:=\tilde{M} \chi_{\Omega}-\hat{M} \chi_{\Omega}$. Then $\operatorname{div} \beta=0$ because $u_{\beta}=0$ a.e. in $\Omega$, where $u_{\beta}$ is calculated from (3.1). Moreover, the only nonzero component of $\beta$ is the $N$ th one. Therefore $\beta=\left(0, \ldots, \beta_{N}\right)$ with $\beta_{N}=\beta_{N}\left(z_{1}, \ldots, z_{N-1}\right)$. As $\beta$ has a compact support we get $\beta=0$ identically. The proposition is proved.

Remark 3.2 If there is a unique representation of $\phi^{* *}$ in terms of a probability measure $\mu_{s}$ on $S^{N-1}$, i.e., if $\phi^{* *}(s)=\int_{S^{N-1}} \sigma \mu_{s}(\mathrm{~d} \sigma)$ for all $s \in \mathbb{B}$, then also (3.10) has a unique solution. This in indeed the case under the assumption of Proposition 3.1. The basic advantage of (3.10) over (3.11) is that in (3.10) we do not work with the convex envelope $\phi^{* *}$. Consequently, the formulation (3.10) can be used even if we do not know an explicit formula for $\phi^{* *}$. 


\subsection{Spatial discretization of (3.10)}

The aim of this subsection is to develop a suitable numerical approximation of the problem (3.10). Besides a discretization of the domain $\Omega$ we will also discretize the support of the Young measure, the sphere $S^{N-1}$. The, simplest but for our purposes sufficient, discretization is to divide $\bar{\Omega}$ into finite volumes $\{\triangle\}_{j=1}^{s}$, $s \in \mathbb{N}$, such that $\bar{\Omega}=\cup_{j=1}^{s} \triangle_{j}$, where $\triangle_{i}$ and $\triangle_{j}$ have disjoint interiors if $i \neq j$. Moreover, we assume that all $\triangle_{j}$ have the same $N$-dimensional Lebesgue measure denoted by $|\triangle|$. Then we will assume that $\nu \in \mathcal{Y}\left(\Omega ; S^{N-1}\right)$ is constant within each finite volume and consists of a finite sum of Dirac masses, saying differently, we suppose that that for any $x \in \triangle_{j}$

$$
\nu_{x}=\sum_{i=1}^{l} \lambda^{j i} \delta_{r^{i}}
$$

with fixed points $r^{i} \in S^{N-1}$ and coefficients $\lambda^{j i}$ satisfying the conditions $0 \leq$ $\lambda^{j i} \leq 1, \sum_{i=1}^{l} \lambda^{j i}=1$ for all $1 \leq j \leq s$ and $1 \leq i \leq l$. Thus, $\nu$ is fully characterized by coefficients $\left\{\lambda^{j i}\right\}$. We refer to [32] for convergence properties of this approximation. As the macroscopic magnetization $M$ is the first moment of $\nu$, we have that $M$ is constant over each $\triangle_{j}$. We denote its value on $\triangle_{j}$ by $M^{j}=\left(M^{j 1}, \ldots, M^{j N}\right)$. Therefore,

$$
M^{j}=\sum_{i=1}^{l} \lambda^{j i} r^{i}, 1 \leq j \leq s .
$$

The demagnetization field energy $\frac{1}{2} \int_{\mathbb{R}^{N}}\left|\nabla u_{M}(z)\right|^{2} \mathrm{~d} z$ is quadratic in $M$ and therefore it is quadratic in $\lambda=\left\{\lambda^{j i}\right\}$. We denote the matrix of the quadratic form assigning $\lambda$ the energy $\int_{\mathbb{R}^{N}}\left|\nabla u_{M}(z)\right|^{2} \mathrm{~d} z$ by $C$. As $M$ is constant over finite volumes, we can work only with spatial averages of the external field $h$. Hence, we put for $1 \leq i \leq T$ and $1 \leq j \leq s$

$$
h_{i}^{j}=\frac{1}{|\triangle|} \int_{\triangle_{j}} h(i, z) \mathrm{d} z .
$$

As a result of this, the discrete anisotropy and external field energies at the time $i$ equal to

$$
\sum_{j=1}^{s}|\triangle| \sum_{t=1}^{l} \lambda_{i}^{j t}\left(\phi\left(r^{k}\right)-r^{k} \cdot h_{i}^{k}\right)
$$

and

$$
D\left(\nu, \nu_{i-1}\right)=\sum_{j=1}^{s} H_{\mathrm{c}}|\triangle|\left|\sum_{k=1}^{l}\left(\lambda^{j k}-\lambda_{i-1}^{j k}\right) r^{k N}\right|
$$


If we denote

$$
\begin{gathered}
\lambda_{i}=\left(\lambda_{i}^{11}, \ldots, \lambda_{i}^{1 l}, \lambda_{i}^{21}, \ldots, \lambda_{i}^{2 l}, \ldots, \lambda_{i}^{s 1} \ldots \lambda_{i}^{s l}\right) \\
a=H_{\mathrm{c}}|\triangle|\left(r^{1 N}, \ldots, r^{l N}\right)
\end{gathered}
$$

and

$$
\begin{aligned}
x= & |\triangle|\left(\phi\left(r^{1}\right)-r^{1} \cdot h_{i}^{1}, \ldots, \phi\left(r^{l}\right)-r^{l} \cdot h_{i}^{1}, \phi\left(r^{1}\right)-r^{1} \cdot h_{i}^{2}, \ldots,\right. \\
& \left.\phi\left(r^{l}\right)-r^{l} \cdot h_{i}^{2}, \ldots, \phi\left(r^{1}\right)-r^{1} \cdot h_{i}^{s}, \phi\left(r^{l}\right)-r^{l} \cdot h_{i}^{s}\right),
\end{aligned}
$$

we see that the discretized version of (3.10) reads: starting with $\lambda_{0}$, find consecutively for $i=1, \ldots, T$ a solution $\lambda_{i}$ of the optimization problem

$$
\operatorname{minimize} \frac{1}{2}\langle C \lambda, \lambda\rangle+\langle x, \lambda\rangle+\sum_{j=1}^{s}\left|\left\langle a, \lambda^{j}-\lambda_{i-1}^{j}\right\rangle\right|
$$

subject to

$$
\begin{aligned}
& B \lambda=b, \\
& \lambda \geq 0,
\end{aligned}
$$

where the constraint $B \lambda=b$ expresses the condition $\sum_{i=1}^{l} \lambda^{j i}=1$ for all $j$.

Although the spatially continuous problem (3.10) has a unique solution for the anisotropy energy considered in Remark 3.2, this is not necessarily the case in (3.13). We can, however, consider the so-called constrained theory proposed in [5], where $\phi$ is considered finite only at two points in $S^{N-1}$ which define the easy-axis of the material. Physically this means that $\phi$ steeply grows from its zero value at magnetic poles. A direct simple remedy consists in a modification of $C$ in (3.13): one adds to $C$ an arbitrarily small multiple of the identity matrix (Prop. 4.1). This way has been used in our numerical tests.

Each optimization problem (3.13) can be equivalently written down as a GE of the type (2.3) which is given by the respective KKT conditions. The state variable $y_{i}$ amounts to the triple $\left(\lambda_{i}, \tau_{i}, \mu_{i}\right)$, where $\tau_{i}$ and $\mu_{i}$ are the multipliers associated with the equality and inequality constraints in (3.13). Hence, one has $\left(\lambda_{i}, \tau_{i}, \mu_{i}\right) \in \mathbb{R}^{n} \times \mathbb{R}^{s} \times \mathbb{R}^{n}$ (so that $\left.k=2 n+s\right), \lambda_{i}=\left(\lambda_{i}^{1}, \lambda_{i}^{2}, \ldots, \lambda_{i}^{s}\right) \in\left(\mathbb{R}^{l}\right)^{s}$ (so that $n=l s$,

$$
F_{i}\left(x, y_{i-1}, y_{i}\right)=F\left(x, \lambda_{i}, \tau_{i}, \mu_{i}\right)=\left[\begin{array}{c}
C \lambda_{i}+x+B^{T} \tau_{i}-\mu_{i} \\
B \lambda_{i}-b \\
\lambda_{i}
\end{array}\right]
$$

and

$$
Q_{i}\left(y_{i-1}, y_{i}\right)=Q\left(\lambda_{i-1}, \lambda_{i}, \mu_{i}\right)=\left[\begin{array}{c}
\partial_{\lambda_{i}} \sum_{j=1}^{s}\left|\left\langle a, \lambda_{i}^{j}-\lambda_{i-1}^{j}\right\rangle\right| \\
0 \\
N_{\mathbb{R}_{+}^{n}}\left(\mu_{i}\right)
\end{array}\right], \quad i=1,2, \ldots, T .
$$


In fact, we do not control our equilibrium model directly via $x$. From (3.12) it follows that $x=\tilde{x}-\hat{x}$, where

$$
\tilde{x}=|\triangle|\left(\phi\left(r^{1}\right), \ldots, \phi\left(r^{l}\right), \phi\left(r^{1}\right), \ldots, \phi\left(r^{l}\right), \ldots, \phi\left(r^{1}\right), \ldots, \phi\left(r^{l}\right)\right) \in\left(\mathbb{R}^{l}\right)^{s}
$$

is the constant vector and

$$
\hat{x}=|\triangle|\left(r^{1} \cdot h_{i}^{1}, \ldots, r^{l} \cdot h_{i}^{1}, r^{1} \cdot h_{i}^{2}, \ldots, r^{l} \cdot h_{i}^{2}, \ldots, r^{1} \cdot h_{i}^{s}, \ldots, r^{l} \cdot h_{i}^{s}\right) .
$$

Considering an external field $h \in \mathbb{R}^{N}$ spatially constant and depending on time through two smooth functions $\alpha_{1}, \alpha_{2}: \mathbb{R} \rightarrow \mathbb{R}$, we infer that $\hat{x}$ depends only on $\alpha_{1}, \alpha_{2}$ and consequently $x=G\left(\alpha_{1}, \alpha_{2}\right)$, where $G: \mathbb{R}^{2} \rightarrow \mathbb{R}^{n}$ is a continuously differentiable function.

Our goal is now to minimize a cost function $\varphi$ depending on $\alpha$ and $\lambda_{T}$. As an example we may suppose that $\varphi$ penalizes a deviation of volume fractions of the resulting magnetization at the time $T$ from a desired value $\bar{\lambda}=\left(\bar{\lambda}^{1}, \ldots, \bar{\lambda}^{s}\right)$. More specifically,

$$
\varphi\left(\alpha, \lambda_{T}\right)=\sum_{j=1}^{s} \beta^{j}\left\|\lambda_{T}^{j}-\bar{\lambda}^{j}\right\|^{2},
$$

where $\beta^{j} \geq 0,1 \leq j \leq s$, represent weight coefficients.

In this way we obtain the MPEEC

$$
\operatorname{minimize} \sum_{j=1}^{s} \beta^{j}\left\|\lambda_{T}^{j}-\bar{\lambda}^{j}\right\|^{2}
$$

subject to

$$
\begin{aligned}
& 0 \in F\left(G\left(\alpha_{1}, \alpha_{2}\right), \lambda_{i}, \tau_{i}, \mu_{i}\right)+Q\left(\lambda_{i-1}, \lambda_{i}\right) \text { with } \lambda_{0} \text { given, } i=1, \ldots, T \\
& \alpha_{1}, \alpha_{2} \in \omega,
\end{aligned}
$$

where $F, Q$ are given in (3.15), (3.16), and $\omega \subset \mathbb{R}^{2}$ is the set of admissible controls $\alpha_{1}, \alpha_{2}$. The next section is devoted to its numerical solution by the implicit programming approach (ImP) developed in Section 2.

\section{Numerical solution}

We start with the verification of assumption (A3).

Proposition 4.1 Let $C$ be positive definite and $\omega^{\prime}$ be an open convex set containing $\omega$. Then the state map $\widehat{S}_{T}:\left(\alpha_{1}, \alpha_{2}\right) \mapsto\left(\lambda_{T}, \tau_{T}, \mu_{T}\right)$ defined via GEs in (3.19) is single-valued and locally Lipschitz for all $\left(\alpha_{1}, \alpha_{2}\right) \in \omega^{\prime}$. 
Proof. The uniqueness of the solution $\lambda_{i}$ to (3.10) has been already mentioned. The uniqueness of the multipliers $\tau_{i}, \mu_{i}$ follows from the fact that, due to the special structure of $B$, the constraint system $B \lambda=b$ and $\lambda \geq 0$ satisfies the linear independence constraint qualification (cf. [26]) at each feasible point.

Let $\widetilde{S}$ be the map which assigns $x$ the unique solution $\left(\lambda_{1}, \tau_{1}, \mu_{1}, \lambda_{2}, \tau_{2}, \mu_{2}, \ldots, \lambda_{T}, \tau_{T}, \mu_{T}\right)$ of the system of GEs

$$
0 \in F\left(x, \lambda_{i}, \tau_{i}, \mu_{i}\right)+Q\left(\lambda_{i-1}, \lambda_{i}\right) \text { with } \lambda_{0} \text { given, } i=1, \ldots, T \text {. }
$$

We observe that $\widetilde{S}$ is polyhedral and hence locally Lipschitz over $\mathbb{R}^{n}$ by virtue of [30], [26, Cor. 2.5]. For the investigated map $\widehat{S}_{T}$ one has

$$
\widehat{S}_{T}=D \circ \widetilde{S} \circ G
$$

where the matrix $D$ realizes the appropriate canonical projection. The Lipschitz continuity of $\widehat{S}_{T}$ on $\omega^{\prime}$ thus follows from the Lipschitz continuity of $G$.

We conclude that all assumptions, needed for the application of ImP to (3.19), are fulfilled and concentrate on the computation of subgradients of the composite objective.

\subsection{Adjoint equation}

The next step consists in the evaluation of $D^{*} Q$ which maps $\mathbb{R}^{n} \times \mathbb{R}^{s} \times \mathbb{R}^{n}$ in subsets of $\mathbb{R}^{n} \times \mathbb{R}^{n} \times \mathbb{R}^{n}$. By elementary coderivative calculus, at a fixed point $\left(\bar{\alpha}, \bar{\lambda}_{i-1}, \bar{\lambda}_{i}, \bar{\tau}_{i}, \bar{\mu}_{i}\right)$ and for $\bar{c}_{i}=\left({ }^{1} \bar{c}_{i}, 0,{ }^{3} \bar{c}_{i}\right)=-F\left(\bar{\alpha}, \bar{\lambda}_{i}, \bar{\tau}_{i}, \bar{\mu}_{i}\right)$, one has

$$
D^{*} Q\left(\bar{\lambda}_{i-1}, \bar{\lambda}_{i}, \bar{\mu}_{i},{ }^{1} \bar{c}_{i}, 0,{ }^{3} \bar{c}_{i}\right)\left({ }^{1} w,{ }^{2} w,{ }^{3} w\right)=\left[\begin{array}{c}
D^{*} P\left(\bar{\lambda}_{i-1}, \bar{\lambda}_{i},{ }^{1} \bar{c}_{i}\right)\left({ }^{1} w\right) \\
0 \\
D^{*} N_{\mathbb{R}_{+}^{n}}\left(\bar{\mu}_{i},{ }^{3} \bar{c}_{i}\right)\left({ }^{3} w\right)
\end{array}\right]
$$

for any $\left({ }^{1} w,{ }^{2} w,{ }^{3} w\right) \in \mathbb{R}^{n} \times \mathbb{R}^{s} \times \mathbb{R}^{n}$. In (4.1), $P$ denotes the partial subdifferential mapping in the first line of (3.16). The coderivative of $N_{\mathbb{R}_{+}^{n}}$ can easily be computed, e.g., on the basis of [25, Lemma 2.2]. The computation of $D^{*} P$ is, however, substantially more demanding. First we observe that, due to a separation of variables,

$$
D^{*} P\left(\bar{\lambda}_{i-1}, \bar{\lambda}_{i},{ }^{1} c_{i}\right)\left({ }^{1} w\right)=\left[\begin{array}{c}
D^{*} P_{1}\left(\bar{\lambda}_{i-1}^{1}, \bar{\lambda}_{i}^{1},{ }^{1} \bar{c}_{i}^{1}\right)\left({ }^{1} w^{1}\right) \\
\vdots \\
D^{*} P_{s}\left(\bar{\lambda}_{i-1}^{s}, \bar{\lambda}_{i}^{s},{ }^{1} \bar{c}_{i}^{s}\right)\left({ }^{1} w^{s}\right)
\end{array}\right]
$$

where ${ }^{1} \bar{c}_{i}=\left({ }^{1} \bar{c}_{i}^{1},{ }^{1} \bar{c}_{i}^{2}, \ldots,{ }^{1} \bar{c}_{i}^{s}\right) \in\left(\mathbb{R}^{l}\right)^{s},{ }^{1} w=\left({ }^{1} w^{1},{ }^{1} w^{2}, \ldots,{ }^{1} w^{s}\right) \in\left(\mathbb{R}^{l}\right)^{s}$ and

$$
P_{j}\left(\lambda_{i-1}^{j}, \lambda_{i}^{j}\right)=\partial_{\lambda_{i}}\left|\left\langle a, \lambda_{i}^{j}-\lambda_{i-1}^{j}\right\rangle\right|, j=1,2, \ldots, s .
$$


Let $\mathcal{M}_{i}\left(\bar{\lambda}_{i-1}, \bar{\lambda}_{i}\right)$ denote the subset of the index set $\{1,2, \ldots, s\}$ such that for $j \in \mathcal{M}_{i}\left(\bar{\lambda}_{i-1}, \bar{\lambda}_{i}\right)$

$$
\left\langle a, \bar{\lambda}_{i}^{j}-\bar{\lambda}_{i-1}^{j}\right\rangle=0
$$

From Definition 1.3 it easily follows that $D^{*} P_{j}\left(\bar{\lambda}_{i-1}^{j}, \bar{\lambda}_{i}^{j},{ }^{1} \bar{c}_{i}^{j}\right)\left({ }^{1} w^{j}\right) \equiv 0$ whenever $j \notin \mathcal{M}_{i}\left(\bar{\lambda}_{i-1}, \bar{\lambda}_{i}\right)$. If $j \in \mathcal{M}_{i}\left(\bar{\lambda}_{i-1}, \bar{\lambda}_{i}\right)$, then we can perform a slight modification of [23, Thm. 3.4] and arrive at the formula

$$
D^{*} P_{j}\left(\bar{\lambda}_{i-1}^{j}, \bar{\lambda}_{i}^{j},{ }^{1} \bar{c}_{i}^{j}\right)\left({ }^{1} w^{j}\right)=\left[\begin{array}{c}
-a \\
a
\end{array}\right] D^{*} \mathfrak{Z}(0, \eta)\left(\left\langle a,{ }^{1} w^{j}\right\rangle\right),
$$

where $\mathfrak{Z}(\cdot)=\partial|\cdot|$, and $\eta \in \partial \mathfrak{Z}(0)=[-1,1]$ is uniquely determined by the equation $\bar{c}_{i}^{j}=a \eta$. One easily verifies that

$$
D^{*} \mathfrak{Z}(0, \eta)(0)=\mathbb{R}
$$

From this it follows that for $j \in \mathcal{M}_{i}\left(\bar{\lambda}_{i-1}, \bar{\lambda}_{i}\right)$

$$
\left(q_{i}^{j},{ }^{1} v_{i}^{j}\right) \in D^{*} P_{j}\left(\bar{\lambda}_{i-1}^{j}, \bar{\lambda}_{i}^{j},{ }^{1} \bar{c}_{i}^{j}\right)\left({ }^{1} w_{i}^{j}\right),
$$

whenever

$$
q_{i}^{j} \in \operatorname{lin} a,{ }^{1} v_{i}^{j}=-q_{i}^{j} \text { and }\left\langle a,{ }^{1} w_{i}^{j}\right\rangle=0 .
$$

These relations can be used together with

$$
\begin{gathered}
q_{i}^{j}={ }^{1} v_{i}^{j}=0 \text { for } j \notin \mathcal{M}_{i}\left(\bar{\lambda}_{i-1}, \bar{\lambda}_{i}\right), \\
{ }^{3} v_{i} \in D^{*} N_{\mathbb{R}_{+}^{n}}\left(\bar{\mu}_{i},{ }^{3} \bar{c}_{i}\right)\left({ }^{3} w_{i}\right)
\end{gathered}
$$

in the adjoint system

$$
\begin{aligned}
0 & =\nabla_{2} \varphi\left(\bar{\alpha}, \bar{y}_{T}\right)+\left[\begin{array}{ccc}
C & B^{T} & -\mathbb{I} \\
B & 0 & 0 \\
E & 0 & 0
\end{array}\right]\left[\begin{array}{l}
{ }^{1} w_{T} \\
{ }^{2} w_{T} \\
{ }^{3} w_{T}
\end{array}\right]+\left[\begin{array}{c}
{ }^{1} v_{T} \\
0 \\
{ }^{3} v_{T}
\end{array}\right] \\
0 & =\left[\begin{array}{c}
q_{T} \\
0 \\
0
\end{array}\right]+\left[\begin{array}{ccc}
C & B^{T} & -\mathbb{I} \\
B & 0 & 0 \\
E & 0 & 0
\end{array}\right]\left[\begin{array}{l}
1 \\
{ }^{2} w_{T-1} \\
{ }^{2} w_{T-1} \\
{ }^{3} w_{T-1}
\end{array}\right]+\left[\begin{array}{c}
{ }^{1} v_{T-1} \\
0 \\
{ }^{3} v_{T-1}
\end{array}\right] \\
& \ldots \ldots \\
0 & =\left[\begin{array}{c}
q_{2} \\
0 \\
0
\end{array}\right]+\left[\begin{array}{ccc}
C & B^{T} & -\mathbb{I} \\
B & 0 & 0 \\
E & 0 & 0
\end{array}\right]\left[\begin{array}{l}
{ }^{1} w_{1} \\
2 \\
{ }^{3} w_{1} \\
{ }^{3} w_{1}
\end{array}\right]+\left[\begin{array}{c}
{ }^{1} v_{1} \\
0 \\
{ }^{3} v_{1}
\end{array}\right] .
\end{aligned}
$$

As a subgradient of the respective function $\Theta$ one can now provide the used bundle method with the vector

$$
\xi=\nabla \varphi\left(\bar{\alpha}, \bar{y}_{T}\right)+\sum_{i=1}^{T}(\nabla G(\bar{\alpha}))^{T} w_{i}^{1} .
$$


To connect relations (4.4), (4.5), (4.6), (4.7) and the adjoint system (4.8), we proceed according to [25, Lemma 2.2] and introduce for each $i=1,2 \ldots, T$ the index sets

$$
\begin{aligned}
I_{i}^{+}\left(\bar{\lambda}_{i}, \bar{\mu}_{i}\right) & :=\left\{j \in\{1,2 \ldots, n\} \mid\left(\bar{\lambda}_{i}\right)^{j}=0,\left(\bar{\mu}_{i}\right)^{j}>0\right\} \\
I_{i}^{0}\left(\bar{\lambda}_{i}, \bar{\mu}_{i}\right) & :=\left\{j \in\{1,2 \ldots, n\} \mid\left(\bar{\lambda}_{i}\right)^{j}=0,\left(\bar{\mu}_{i}\right)^{j}=0\right\} \\
I_{i}^{f}\left(\bar{\lambda}_{i}, \bar{\mu}_{i}\right) & :=\left\{j \in\{1,2 \ldots, n\} \mid\left(\bar{\lambda}_{i}\right)^{j}>0,\left(\bar{\mu}_{i}\right)^{j}=0\right\}
\end{aligned}
$$

related to the inequality constraints $\left(\lambda_{i}\right)^{j} \geq 0, j=1,2, \ldots, n$. We take any partitioning of $I_{i}^{0}\left(\bar{\lambda}_{i}, \bar{\mu}_{i}\right)$ :

$$
I_{i}^{0}\left(\bar{\lambda}_{i}, \bar{\mu}_{i}\right)={ }^{1} \beta_{i} \cup{ }^{2} \beta_{i}
$$

and define

$$
\widetilde{I}_{i}^{+}:=I_{i}^{+}\left(\bar{\lambda}_{i}, \bar{\mu}_{i}\right) \cup{ }^{1} \beta_{i} \quad \widetilde{I}_{i}^{f}:=I_{i}^{f}\left(\bar{\lambda}_{i}, \bar{\mu}_{i}\right) \cup{ }^{2} \beta_{i} .
$$

Consider now the equation system

$$
\left[\begin{array}{cccc}
C & B^{T} & -\mathbb{I}_{\tilde{I}_{i}^{+}} & A_{\mathcal{M}_{i}} \\
B & 0 & 0 & 0 \\
\left(\mathbb{I}_{\widetilde{I}_{i}^{+}}\right)^{T} & 0 & 0 & 0 \\
\left(A_{\mathcal{M}_{i}}\right)^{T} & 0 & 0 & 0
\end{array}\right]\left[\begin{array}{c}
{ }^{1} w_{i} \\
{ }^{2} w_{i} \\
{ }^{3} \tilde{w}_{i} \\
\tilde{v}_{i}
\end{array}\right]=\left[\begin{array}{c}
\tilde{q}_{i} \\
0 \\
0 \\
0
\end{array}\right]
$$

where $A$ is the $(n \times s)$ matrix, defined by

$$
A:=\left[\begin{array}{cccc}
a & 0_{l \times 1} & \cdots & 0_{l \times 1} \\
0_{l \times 1} & a & \cdots & 0_{l \times 1} \\
& & \ddots & \\
0_{l \times 1} & 0_{l \times 1} & \cdots & a
\end{array}\right]
$$

and ${ }^{3} \tilde{w}_{i}$ is a subvector of ${ }^{3} w_{i}$, whose components belong to $\tilde{I}_{i}^{+}\left(\bar{\lambda}_{i}, \bar{\mu}_{i}\right)$. For notational simplicity, we occasionally omit the arguments of $\mathcal{M}_{i}, I_{i}^{+}, I_{i}^{0}$ and $I_{i}^{f}$.

This system has to be solved backwards for $i=T, T-1, \ldots, 1$ with the terminal condition $\tilde{q}_{T}=-\nabla_{2} \varphi\left(\bar{\alpha}, \bar{\lambda}_{T}\right)$ by using the updates

$$
\begin{gathered}
\left(\tilde{q}_{i}\right)_{\mathcal{M}_{i}}=\tilde{v}_{i} \\
\left(\tilde{q}_{i}\right)^{j}=0 \text { for } j \notin \mathcal{M}_{i}\left(\bar{\lambda}_{i-1}, \bar{\lambda}_{i}\right),
\end{gathered}
$$

$i=T-1, T-2, \ldots, 1$. One can easily verify that the component ${ }^{1} w_{i}$ to each solution of (4.11) is feasible also with respect to (4.4), (4.5), (4.6), (4.7) and the adjoint system (4.8). It may thus be used in formula (4.9). Note that the sizes of $\tilde{v}_{i}$ and ${ }^{3} \tilde{w}_{i}$ vary, depending on the cardinality of $\mathcal{M}_{i}\left(\bar{\lambda}_{i-1}, \bar{\lambda}\right)$ and $\tilde{I}_{i}^{+}$, respectively.

Remark 4.2 The choice of the partitioning (4.10) has an essential influence on which subgradient we actually compute. As already mentioned, we may even 
compute a vector that does not belong to $\partial \Phi$. This was indeed the case in one of our numerical examples. We considered a problem with only one design variable. The data were set so that the composite objective was constant in a neighborhood of $\alpha^{\prime}$ and that $I_{i}^{0} \neq \emptyset$ at $\alpha^{\prime}$. Still, when choosing ${ }^{1} \beta_{i}=\emptyset$, i.e., $\widetilde{I}_{i}^{+}=I_{i}^{+}$, we have obtained a nonzero subgradient at $\alpha^{\prime}$. A simple remedy to this unpleasant situation is to change the partitioning (4.10); we know that there is a partitioning that leads to a true subgradient. In our particular case, we have taken ${ }^{2} \beta_{i}=\emptyset$, i.e., $\widetilde{I}_{i}^{+}=I_{i}^{+} \cup I_{i}^{0}$. For this choice, we got a subgradient equal to zero, a correct one.

\subsection{Computational procedures}

Numerical solution of the MPEEC (3.19) requires solution of several subproblems. First, for a given control variable, we have to solve the state problem: a series of nonsmooth convex optimization problems (3.13). We transform it by a simple trick to a convex quadratic program. By introducing an auxiliary variable $z \in \mathbb{R}^{s}$ we can write (3.13) equivalently as

$$
\min _{\lambda, z} \frac{1}{2}\langle C \lambda, \lambda\rangle+\langle x, \lambda\rangle+\sum_{j=1}^{s} z^{j}
$$

subject to $\lambda \geq 0$

$$
\begin{aligned}
& B \lambda=b \\
& z^{j} \geq\left\langle a, \lambda^{j}-\lambda_{i-1}^{j}\right\rangle, \quad j=1, \ldots, s \\
& z^{j} \geq-\left\langle a, \lambda^{j}-\lambda_{i-1}^{j}\right\rangle, \quad j=1, \ldots, s .
\end{aligned}
$$

This problem can be solved by any QP solver; we have opted for the code PENNON that proved to be very efficient for general (in particularly convex) nonlinear programming problems [12].

Second, we must solve the adjoint systems (4.11). These are medium-size systems of linear equations with nonsymmetric matrices. The matrices, however, may be singular in case $\mathcal{M}_{i}^{0} \neq \emptyset$. When we know that the matrix is nonsingular (i.e., when $\mathcal{M}_{i}^{0}=\emptyset$ ), we use the LAPACK subroutine DGESV based on LU decomposition with partial pivoting; in the general case, we use the least-square subroutine DGELSD. Note that even for the nonsingular matrix, the first component of the solution ${ }^{1} w_{i}$ is always unique - and this is the only component we need.

Finally, we have to minimize the respective composite objective $\Theta$ over $\omega$. It was already mentioned that this is a nonsmooth, nonconvex and Lipschitz function. We have used one of the few suitable codes for minimization of such functions, the code BT [36]. 


\subsection{Examples}

The examples presented in this section are purely academic. Their purpose is to verify that the proposed technique can be successfully used for solving MPEECs. On the other hand, it is not difficult to modify these problems to real-world applications, by changing the sample geometry, level of discretization and, in particular, the cost function and the design variables.

We consider $N=2, \phi\left(r_{1}, r_{2}\right)=30 r_{1}^{2}$, a sample of dimensions $2 \times 1$, spatial discretization by $8 \times 8$ finite volumes and 10 time-steps. We further set $l=4$ (the discretization of the sphere $S^{N-1}$; see Section 3.2).

The control variables are the amplitude and frequency of the second component of the external magnetic field $h_{i}$ (independent of spatial variable). We consider $h_{i}=\left(0, f_{i}\right)$ with

$$
f_{i}=\alpha_{1} \sin \left(\frac{2 \pi}{\alpha_{2}} \frac{i}{T}\right), \quad i=1,2, \ldots, T .
$$

This, by (3.17), defines the function $G$.

The admissible control set $\omega$ is $\left[0.1,10^{5}\right] \times\left[0.1,10^{5}\right]$.

Example 1 In the first example we try to magnetize fully the specimen in $\mathbb{R}^{2}$ in the second direction, so that the desired magnetization $M$ is $(0,1)$. This can be done by setting $\bar{\lambda}^{j 1}=1.1, j=1, \ldots, s$ (note that the maximal possible value of $\lambda$ is equal to one). The initial value of $\alpha$ is set to $(50,4)$. The problem appears to be "easy" and BT finds the optimum in just a few steps. Below we show the output of the code:

$\begin{array}{ccccc}\begin{array}{l}\text { BT-Algorithm } \\ \text { ========== }\end{array} & & & \\ \text { niter } & \text { ncomp } & f & \text { gp } & \text { alpha } \\ 1 & 1 & 0.43362311 \mathrm{E}+00 & 0.67345917 \mathrm{E}-01 & 0.00000000 \mathrm{E}+00 \\ 2 & 2 & 0.43362311 \mathrm{E}+00 & 0.53359109 \mathrm{E}-01 & 0.10000000 \mathrm{E}-01 \\ 3 & 3 & 0.38786221 \mathrm{E}+00 & 0.56068671 \mathrm{E}-01 & 0.12958132 \mathrm{E}-01 \\ 4 & 4 & 0.32000000 \mathrm{E}+00 & 0.17855778 \mathrm{E}-15 & 0.00000000 \mathrm{E}+00\end{array}$

We can see that the exact minimum $\lambda_{T}^{j 1}=1, j=1, \ldots, s / 2$, (giving the optimal objective value $f^{*}=32 \cdot(1.0-1.1)^{2}$ ) was found in just four steps. The reason for that is simple: from a certain value of the amplitude $\alpha_{1}^{\prime}$ up (for a given frequency), the cost function is constant (and optimal) - the specimen is fully magnetized and any increase of the amplitude cannot change it. The BT code then quickly hits any $\alpha_{1}>\alpha_{1}^{\prime}$ and finishes.

Due to the simplicity of the example, it is not surprising that we obtain the same result when we change the time discretization, even to just one time step. Only the BT iterates will be slightly different. It is, however, interesting to 


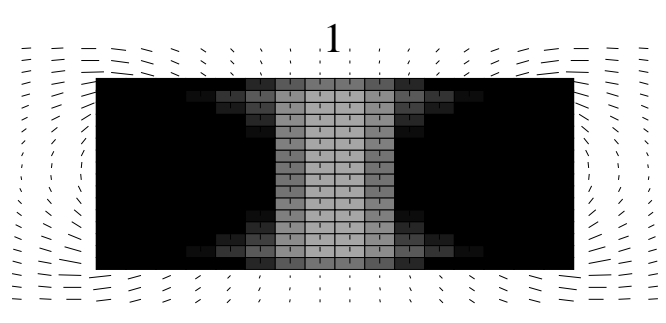

3

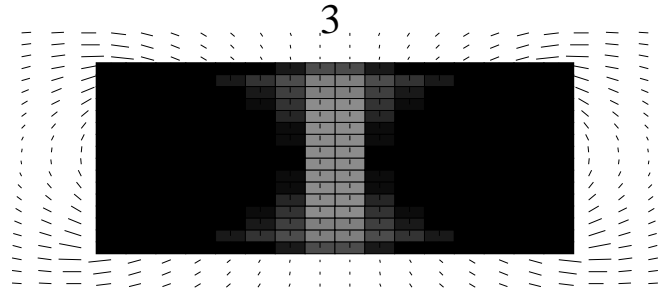

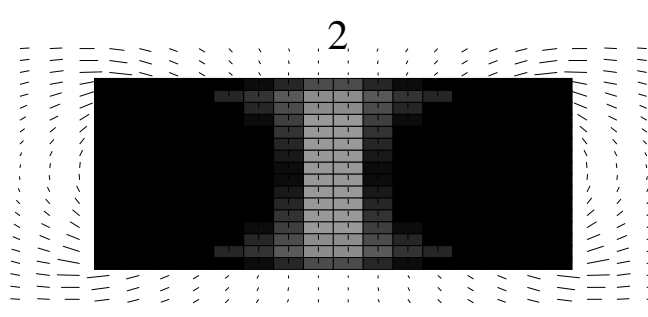

4

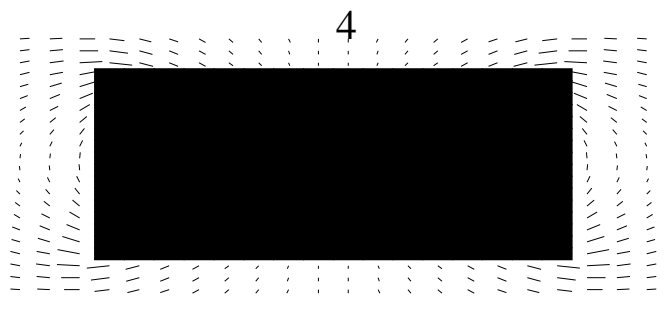

Figure 1: The specimen, its magnetization, and the demagnetizing field $\nabla u_{M}$ around the specimen in each iteration of BT. The darker is the color in a point of the specimen the closer is the magnetization to $(0,1)$ (in the Euclidean norm).

note that, in each iteration (and for any time discretization), only one time step contributed to the second term of the subgradient (4.9); that means, $w_{i}^{1} \neq 0$ for only one $i \in\{1, \ldots, T\}$, whereas this $i$ was different at every BT iterate.

Example 2 In the second example, we set $\bar{\lambda}^{j 1}=0.9, j=1, \ldots, s$, and try to identify this value. This time, the objective function is not constant around the optimum and the behavior of the BT code reminds more a standard behavior of a minimization method:

$\begin{array}{ccccc}\begin{array}{c}\text { BT-Algorithm } \\ =========\end{array} & & & \\ \text { niter } & \text { ncomp } & f & \text { gp } & \text { alpha } \\ 1 & 1 & 0.25601552 \mathrm{E}+00 & 0.24693051 \mathrm{E}-01 & 0.00000000 \mathrm{E}+00 \\ 2 & 2 & 0.25601552 \mathrm{E}+00 & 0.24693051 \mathrm{E}-01 & 0.00000000 \mathrm{E}+00 \\ 3 & 3 & 0.23502032 \mathrm{E}+00 & 0.24693051 \mathrm{E}-01 & 0.10000000 \mathrm{E}-01 \\ 4 & 4 & 0.17673858 \mathrm{E}+00 & 0.20989044 \mathrm{E}-01 & 0.00000000 \mathrm{E}+00 \\ 5 & 5 & 0.17673858 \mathrm{E}+00 & 0.17128780 \mathrm{E}-01 & 0.69166119 \mathrm{E}-02 \\ 6 & 6 & 0.17673858 \mathrm{E}+00 & 0.11764406 \mathrm{E}-01 & 0.91444459 \mathrm{E}-02 \\ 7 & 7 & 0.16785966 \mathrm{E}+00 & 0.58038202 \mathrm{E}-02 & 0.00000000 \mathrm{E}+00 \\ 8 & 8 & 0.16652221 \mathrm{E}+00 & 0.35283823 \mathrm{E}-02 & 0.23490041 \mathrm{E}-03 \\ 9 & 10 & 0.16652221 \mathrm{E}+00 & 0.16319657 \mathrm{E}-02 & 0.12254303 \mathrm{E}-03 \\ 10 & 11 & 0.16643681 \mathrm{E}+00 & 0.58649082 \mathrm{E}-03 & 0.00000000 \mathrm{E}+00 \\ 11 & 12 & 0.16643681 \mathrm{E}+00 & 0.29875680 \mathrm{E}-03 & 0.31325080 \mathrm{E}-05 \\ 12 & 13 & 0.16643417 \mathrm{E}+00 & 0.10889954 \mathrm{E}-03 & 0.00000000 \mathrm{E}+00 \\ 13 & 14 & 0.16643417 \mathrm{E}+00 & 0.54258298 \mathrm{E}-04 & 0.10805660 \mathrm{E}-06 \\ 14 & 15 & 0.16643408 \mathrm{E}+00 & 0.20402428 \mathrm{E}-04 & 0.00000000 \mathrm{E}+00\end{array}$


1

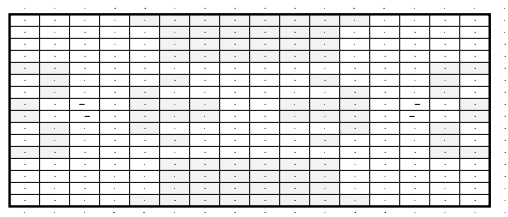

3

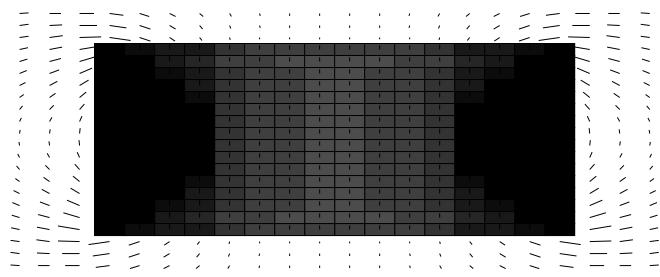

2

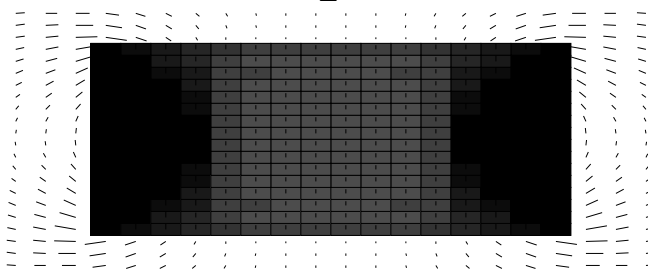

4

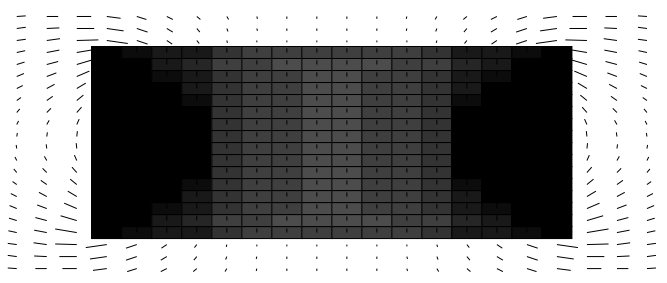

Figure 2: The specimen, its magnetization, and the demagnetizing field $\nabla u_{M}$ around the specimen in each 5th iteration of BT. The darker is the color in a point of the specimen the closer is the magnetization to $(0,1)$ (in the Euclidean norm).

\begin{tabular}{rrrrr}
15 & 16 & $0.16643408 \mathrm{E}+00$ & $0.10207925 \mathrm{E}-04$ & $0.37928982 \mathrm{E}-08$ \\
16 & 17 & $0.16643408 \mathrm{E}+00$ & $0.38203558 \mathrm{E}-05$ & $0.00000000 \mathrm{E}+00$ \\
17 & 18 & $0.16643408 \mathrm{E}+00$ & $0.19099423 \mathrm{E}-05$ & $0.13298890 \mathrm{E}-09$ \\
18 & 19 & $0.16643408 \mathrm{E}+00$ & $0.71546057 \mathrm{E}-06$ & $0.00000000 \mathrm{E}+00$ \\
19 & 20 & $0.16643408 \mathrm{E}+00$ & $0.35774567 \mathrm{E}-06$ & $0.46642216 \mathrm{E}-11$ \\
20 & 21 & $0.16643408 \mathrm{E}+00$ & $0.13400225 \mathrm{E}-06$ & $0.00000000 \mathrm{E}+00$ \\
21 & 22 & $0.16643408 \mathrm{E}+00$ & $0.67024577 \mathrm{E}-07$ & $0.16361832 \mathrm{E}-12$ \\
\multicolumn{2}{l}{ convergence } & & &
\end{tabular}

However, the minimal point $(45.17065468,4.264825646)$ is not unique, again. When we fix the value of $\alpha_{1}$ to 50.0 and keep only $\alpha_{2}$ free, BT finds a point $(50.0,5.619174535)$ with the same value of the objective function.

\section{Conclusion}

We formulated a problem of optimal control of a ferromagnet in a form of an MPEEC and developed a solution approach based on an implicit programming technique. The adjoint equations, needed to compute the subgradients of the composite objective, are derived using the generalized differential calculus of B. Mordukhovich. Up to our knowledge, this is the first attempt to solve mathematical programs with evolutionary equilibria numerically. Computational tests demonstrate the applicability of this approach.

The implicit programming technique requires local uniqueness of the equilibrium problem. To enforce uniqueness, we modified slightly the matrix $C$ in 
(3.13). If, however, the cost functions $\varphi$ depends only on the spatial average of the magnetization over the specimen, i.e., on $|\Omega|^{-1} \int_{\Omega} M(z) \mathrm{d} z$ or on the selfinduced magnetic field $\nabla u_{M}$ then, as these quantities are uniquely defined even in the discrete unconstrained case (3.13) [4], assumption (A3) holds automatically. Hence, the control problems may be to find an external field $h$ such that the average magnetization is as "close" as possible to a given vector and similarly for the self-induced field. This demonstrates a wider applicability of our results than the examples solved in this contribution.

\section{Acknowledgment}

This research was supported by the Academy of Sciences of the Czech Republic through grant No. A1075402. M.K. was also supported by the IMA at the University of Minnesota, Minneapolis, during his stay in November 2004. The authors would like to thank Miloš Jirsa (Institute of Physics, Academy of Sciences of the Czech Republic) for a fruitful discussion.

\section{References}

[1] J. P. Aubin and H. Frankowska. Set-Valued Analysis. Birkhäuser, Boston, 1990.

[2] W. F. Brown, Jr. Magnetostatic principles in ferromagnetism. Springer, New York, 1966.

[3] S. Dempe. Foundations of Bilevel Programming. Kluwer Acad. Publ., Dordrecht-Boston-London, 2002.

[4] A. DeSimone. Energy minimizers for large ferromagnetic bodies. Arch. Rat. Mech. Anal., 125:99-143, 1993.

[5] A. DeSimone and R. D. James. A constrained theory of magnetoelasticity. J. Mech. Phys. Solids, 50:283-320, 2002.

[6] R. Henrion, A. Jourani, and J. Outrata. On the calmness of a class of multifunctions. SIAM Journal on Optimization, 13(2):603-618, 2002.

[7] A. Hubert and R. Schäffer. Magnetic Domains. Springer, Berlin, 1998.

[8] R. D. James and D. Kinderlehrer. Frustration in ferromagnetic materials. Continuum Mech. Thermodyn., 2:215-239, 1990.

[9] M. Kočvara, A. Mielke, and T. Roubíček. Rate-independent approach to the delamination problem. Preprint 2003/29, SFB 404 "Mehrfeldprobleme 
in der Kontinuumsmechanik", Universität Sttutgart, 2003. To appear in Mathematics and Mechanics of Solids.

[10] M. Kočvara and J. V. Outrata. On the modeling and control of delamination processes. In J. Cagnol and J.-P. Zolesion, editors, Control and Boundary Analysis, pages 171-190. Marcel Dekker, New York, 2004.

[11] M. Kočvara and J. V. Outrata. Optimization problems with equilibrium constraints and their numerical solution. Mathematical Programming B, 101:119-150, 2004.

[12] M. Kočvara and M. Stingl. PENNON - a code for convex nonlinear and semidefinite programming. Optimization Methods and Software, 18:317-333, 2003 .

[13] M. Kružík. Maximum principle based algorithm for hysteresis in micromagnetics. Adv. Math. Sci. Appl., 13:461-485, 2003.

[14] M. Kružík. Periodic solutions to a hysteresis model in micromagnetics. IMA preprint 1946/2003, University of Minnesota, Minneapolis., 2003.

[15] M. Kružík. Periodicity properties of solutions to a hysteresis model in micromagnetics. In M. Feistauer et al., editor, Proceedings of ENUMATH 2003 the 5th European Conference on Num. Math. and Adv. Appl., pages 605-614, Springer, Berlin, 2004.

[16] L. D. Landau and E. M. Lifshitz. On the theory of the dispersion of magnetic permeability of ferromagnetic bodies. Physik Z. Sowjetunion, 8:153-169, 1935 .

[17] Z.-Q. Luo, J.-S. Pang, and D. Ralph. Mathematical Programs with Equilibrium Constraints. Cambridge University Press, Cambridge, 1996.

[18] M. Luskin and L. Ma. Analysis of the finite element approximation of microstructure in micromagnetics. SIAM J. Num. Anal., 29:320-331, 1992.

[19] A. Mielke. Energetic formulation of multiplicative elastoplasticity using dissipation distances. Continuum Mech. Thermodyn. To appear.

[20] A. Mielke and F. Theil. Mathematical model for rate-independent phase transformations. In H.-D. Alber, R. Balean, and R.Farwig, editors, Models of Cont. Mechanics in Analysis and Engineering, pages 117-129. ShakerVerlag, Aachen, 1999.

[21] A. Mielke and F. Theil. On rate-independent hysteresis models. Nonlin. Diff. Eq. Appl., 11:151-189, 2004. 
[22] B. S. Mordukhovich. Lipschitzian stability of constraint systems and generalized equations. Nonlinear Anal.- Th. Meth. Appl., 22:173-206, 1994.

[23] B. S. Mordukhovich and J. V. Outrata. On second-order subdifferentials and their applications. SIAM J. Optimization, 12:139-169, 2001.

[24] Boris S. Mordukhovich. Generalized differential calculus for nonsmooth and set-valued mappings. Journal of Mathematical Analysis and Applications, (183):250-288, 1994.

[25] J. V. Outrata. Optimality conditions for a class of mathematical programs with equilibrium constraints. Math. Oper. Res., 24:627-644, 1999.

[26] J. V. Outrata, M. Kočvara, and J. Zowe. Nonsmooth Approach to Optimization Problems with Equilibrium Constraints: Theory, Applications and Numerical Results. Kluwer Acad. Publ., Dordrecht-Boston-London, 1998.

[27] P. Pedregal. Relaxation in ferromagnetism: the rigid case. J. Nonlin. Sci., 4:105-125, 1994.

[28] P. Pedregal. Parametrized Measures and Variational Principles. Birkhäuser, Basel, 1997.

[29] S. M. Robinson. Strongly regular generalized equations. Mathematics of Operations Research, 5:43-62, 1980.

[30] S. M. Robinson. Some continuity properties of polyhedral multifunctions. Mathematical Programming Study, 14:206-214, 1981.

[31] R. T. Rockafellar and R. Wets. Variational Analysis. Springer Verlag, Berlin, 1998.

[32] T. Roubíček. Relaxation in Optimization Theory and Variational Calculus. W. de Gruyter, Berlin, 1997.

[33] T. Roubíček and M. Kružík. Microstructure evolution model in micromagnetics. Zeitschrift f. Angew. Math. Phys., 55:159-182, 2004.

[34] T. Roubíček and M. Kružík. Mesoscopic model for ferromagnets with isotropic hardening. Zeitschrift f. Angew. Math. Phys., 56:107-135, 2005.

[35] S. Scholtes. Introduction to Piecewise Differential Equations. Habilitation Thesis, Institut für Statistik und Mathematische Wirtschaftstheorie, Universität Karlsruhe, Germany, 1994.

[36] H. Schramm and J. Zowe. A version of the bundle idea for minimizing a nonsmooth function: conceptual idea, convergence analysis, numerical results. SIAM J. Optimization, 2:121-152, 1992. 
[37] A. Visintin. A Weiss-type model of ferromagnetism. Physica B, 275:87-91, 2000.

[38] L. C. Young. Generalized curves and existence of an attained absolute minimum in the calculus of variations. Comptes Rendus de la Société et des Lettres de Varsovie, Classe III, 30:212-234, 1937. 